\title{
A integralidade da atenção em diabéticos com doença periodontal
}

\author{
The integrality of the attention in diabetics with periodontal disease
}

\author{
Aline M endes Silva ${ }^{1}$ \\ Andréa M aria D uarte Vargas ${ }^{2}$ \\ EfigêniaFerreira eFerreira ${ }^{2}$ \\ M auro Henrique N ogueira Guimarães de Abreu²
}

\footnotetext{
${ }^{1}$ Coordenação deSaúde Bucal, Secretaria M unicipal deSaúde de Contagem. Rua General David Sarnoff 3.313, Inconfidentes. 32210-110 Contagem M G. mendes.silva.aline@gmail.com ${ }^{2}$ Faculdade de $\mathrm{O}$ dontologia, UniversidadeFederal de M inas Gerais.
}

Abstract The aim of this study was to evaluate the periodontal conditions of individuals with diabetes and to analyze how SU S has contributed to the health attention of these people in Belo H orizonte, M inas Gerais State. For that, a sample of 300 individuals was selected and interviews with diabetics and health unit managers were conducted. From those, $55 \%$ presented gingivitis, $35.3 \%$ periodontitis and $9.7 \%$ were healthy. As for the integral attention for diabetics in SU S, it was observed that despite the fact that most of them wereunder medical supervision, only $27.3 \%$ were under dental treatment in basic health care units, 3.6\% had specialized dental care and only $3.4 \%$ were seen by other health workers. Interdisciplinary care and attention in all levels of the system are essential factors for the integrality of health actions.

Key words Integrality, Periodontal disease, Diabetes mellitus, Oral health, Integral health care
Resumo 0 objetivo deste estudo foi avaliar como está organizado 0 atendimento aos indivíduos com diabetes melito, usuários do SUS, a partir dos dados das condições periodontais apresentadas por este grupo, em Belo Horizonte (M G). Para tanto, uma amostra representativa de trezentos indivíduos foi selecionada para avaliação clínica e entrevista. Foram realizadas entrevistastambém com os gerentes de unidades de saúde. Dos avaliados, $55 \%$ apresentaram gengivite, $35,3 \%$, periodontite e 9,7\% eram saudáveis. Em relação à integralidade da atenção ao diabético no SUS, pôde-se observar que, apesar da maioria estar sob acompanhamento médico, somente $27,3 \%$ estavam sob tratamento odontológico na redebásica, 3,6\% recebiam atendimento especializado em odontologia e apenas 3,4\% eram atendidos por outros profissionais da saúde. 0 atendimento interdisciplinar ea atenção em todos os níveis do sistema são fatores essenciais para a integralidade das ações em saúde. Palavras-chave Integralidade, D oença periodontal, Diabetes melito, Saúdebucal, Atenção integral à saúde 
Introdução

A integralidade da atenção possui como repre sentante histórico mais significativo a medicina integral, movimento surgido nos Estados Unidos da América em resposta à fragmentação da medicina proposta pelo modelo flexneriano, que ressal tava os aspectos biológicos do ser humano em detrimento dos aspectos psicológicos e sociais. A necessidade dese resgatar a percepção do todo e, assim, melhor atender às necessidades da população, desencadeou este movimento ${ }^{1}$.

$\mathrm{Na}$ literatura, diversos autores procuram estabelecer definições do termo integralidade da atenção à saúde. Para $M$ attos ${ }^{1}$, a integralidade poderia ser definida em três conjuntos de sentidos. 0 primeiro estaria relacionado à visão integral do profissional desaúdeem relação ao usuário do serviço. 0 segundo seria referente às formas de organizar as práticas e processos de trabalho em saúde, que deveriam articular as ações de promoção, prevenção, assistência e recuperação da saúde. 0 terceiro estaria relacionado às políticas especiais de saúde, com o estabelecimento deintegração entreos diferentes níveis deatenção e a estruturação de sistemas de referência e contrarreferência, objetivando 0 atendimento integral dos usuários.

A discussão sobre a integralidade da atenção no Brasil surge como uma das principais bandeiras de luta da Reforma Sanitária nos anos se tenta, em busca de um sistema de saúde mais justo e universal ${ }^{2}$. Na década de oitenta, debates importantes foram travados no campo da Saúde Coletiva e da medicina comunitária em busca de valores a serem sustentados pelos profissionais de saúde em termos de suas práticas ${ }^{1}$. A integralidade aparece como um diferencial no Brasil, com a proposta da construção deum Sistema Ú nico deSaúde(SUS) quecontemplasse as ações desaúde de forma integrada e articulada entre os diferentes níveis do sistema ${ }^{3}$.

Desde sua implantação, vários progressos têm sido alcançados no desenvolvimento da atenção básica à saúde. As unidades de saúde destinadas a prestar serviços à população experimentaram um crescimento bastante expressivo, tanto em número absoluto quanto em produção de serviços e aumento de cobertura assistencial em áreas antes desassistidas4. A pesar dos avanços obtidos com o SUS, seus princípios orientadores ainda não são realidade no cotidiano dos serviços desaúde 5 . Dentreseus princípios ediretrizes, talvez a integralidade seja o menos visível na trajetória do sistema e de suas práticas. Isto porque a criação do SU S modificou o arranjo institucional, mas, não necessariamente, transformou as práticas dos serviços de modo a articular a prevenção e a assi stência ${ }^{5}$. Nas unidades básicas, 0 trabalho tem sido organizado, tradicionalmente, de forma fracionada, em função da consolidação das corporações profissionais nos serviços, pela especialização do saber e, consequentemente, do trabalho em saúde ${ }^{6}$.

No contexto da integralidade, faz-senecessária uma reflexão crítica sobre os processos de trabalho em saúde visando à produção de novos conhecimentos e ao desenvolvimento de novas práticas de saúde consoantes com os princípiose diretrizes do SUS. N esteescopo de reflexões, deve ser incluída a aten ção à saúde prestada ao indivíduo com diabetes melito (DM), em vista à complexidade exigida no seu atendimento e, portanto, necessidade da integração dos profissionais que constituem as equipes de saúde das unidades básicas eespecial izadas do SU S. Além disto, a inserção de outros profissionais como nutricionistas, assistentes sociais, psicólogos, professores de educação física é fundamental para o desenvolvimento deuma ação interdisciplinar eintegral na preven ção etratamento do $\mathrm{DM}^{7}$. A prestação de um atendimento integral a este grupo é de suma relevância, uma vez que o DM configura um problema de saúde publica mundial e nacional - 140 milhões de pessoas afetadas em todo mundo e cinco milhões no Brasil7.

Com o propósito de reorganizar a rede de saúde, com melhoria da atenção aos portadores deDM e estabelecer diretrizes e metas para a reorganização da atenção a estesindivíduos no SUS, o Ministério da Saúde (MS) lançou o Plano de Reorganização da Atenção à H ipertensão Arterial eao $\mathrm{DM}^{8}$. 0 objetivo deste plano foi garantir 0 diagnóstico, a vinculação do indivíduo às unidades de saúde para tratamento e acompanhamento, a reestruturação ea ampliação do atendimento resolutivo e de qualidade para os portadores dessas patologias na rede pública de serviços de saúde $^{3}$. A pesar da importância das ações implementadas pelo $\mathrm{MS}$, em relação à atenção à saúde dos indivíduos com DM , observa-se que as ações em saúde bucal não foram incluídas no elenco de propostas para garantir o controle e melhoria da qualidade de vida destegrupo. D estaca-se aqui a relação de risco estabelecida na literatura entre o DM e a doença periodontal 9 .

Estudos sugerem que o DM pode acelerar a destruição periodontal e que, em contrapartida, o tratamento periodontal pode reduzir as exigências de insulina emelhorar o equilíbrio meta- 
bólico do indivíduo com DM. Portanto, os diabéticos precisam de tratamento e controle da doença periodontal9,10. N esta perspectiva, a participação efetiva da equipe de saúde bucal no apoio ao cuidado aos usuários que apresentam doenças crônicas como o DM aponta para uma abordagem voltada aos princípios da integralidade. Este trabalho pretende avaliar como está organizado 0 atendimento aos indivíduos com diabetes melito, usuários do SUS, a partir doS dados das condições periodontais apresentadas por este grupo, em Belo Horizonte.

\section{Métodos}

0 presenteestudo foi submetido eaprovado pelo Comitê de Ética em Pesquisa da Universidade Federal de M inas Gerais (UFM G) e pelo Comitê de Ética e Pesquisa da Secretaria Municipal de Saúde de Belo Horizonte ${ }^{11}$.

0 trabalho foi desenvolvido nas unidades básicas de saúde (UBS) do município de Belo H orizonte (MG), distribuídas pel os nove distritos sanitários que compõem a divisão políticoadministrativa do município. Em cada distrito, foram sorteadas duas UBS para a realização da coleta de dados.

O estudo compreendeu duas etapas: na primeira, foi realizado um estudo epidemiológico em que se utilizou uma amostra representativa de indivíduos diabéticos para determinar a prevalência egravidade da doença periodontal nesta população. $\mathrm{Na}$ segunda etapa, foram realizadas entrevistas estruturadas com estes indivíduos e com os gerentes das U BS estudadas, para conhecer a organização e rotina de atendimento dos diabéticos nas UBS.

Para o cálculo da amostra dos pacientes com DM , utilizou-seo método deestimativa deproporção populacional para a ocorrência de doenças, da Organização Mundial de Saúde (OMS) ${ }^{12}$. Considerando um nível de significância de $95 \%$, erro de $5 \%$ ea prevalência de doença periodontal em diabéticos de $75 \%{ }^{13}$, seria necessário avaliar 288 indivíduos. Para prevenir possíveis perdas, a pesquisa foi concluída com uma amostra final de trezentos indivíduos diabéticos, de ambos os sexos.

A partir do total de indivíduos diabéticosatendidos em cada distrito sanitário, verificado no cadastro do HIPERDIA (sistema informatizado que permite cadastrar e acompanhar os portadores de hipertensão arterial e/ou diabetes melito em todas as unidades ambulatoriais do SUS) ${ }^{14}$, obteve-se a proporcionalidade da amostra, por distrito. Os indivíduos participantes foram sorteados al eatoriamente entre aqueles cadastrados nas U BS até que se obtivesse um número de exames necessários a cada unidade. Considerou-se como critérios deinclusão o indivíduo diabético, dentado, inscrito no HIPERDIA/SUS/BH e com mais de trinta anos.

Para a coleta dos dados epidemiológicos, foi realizado um estudo piloto, com objetivo de realizar a calibração intraexaminador, testar os instrumentos e para treinamento do pesquisador. Os exames de calibração tiveram um intervalo de sete dias entre o primeiro e o segundo exame. U m total de 588 superfícies foram avaliadas e reavaliadas para que a concordância pudesse ser determinada. A plicou-seo teste de concordância Kappa (K), encontrando valores considerados satisfatórios para profundidade de sondagem $(0,81)$, perda de inserção clínica $(0,91)$ e sangramento à sondagem $(0,79)$.

0 roteiro das entrevistas foi testado em um segundo estudo piloto, envolvendo dez usuários e o gerente de uma unidade de saúde, não incluídos no estudo principal. Alguns pequenos ajustes foram realizados no roteiro das entrevistas e na ficha clínica com objetivo de adequar melhor os instrumentos a serem utilizados no estudo principal.

Os exames clínicos foram realizados em consultório odontológico, utilizando o refletor para iluminação, seringa tríplice para secagem dos dentes, espel hos bucais, gazes e sonda periodontal tipo Willians (Hu-Friedy ${ }^{\circledR}$, respeitando-seas normas de biossegurança ${ }^{15}$. A presença de doença periodontal foi avaliada através dos parâmetros clínicos: profundidade de sondagem, perda de inserção clínica e sangramento à sondagem. Para determinação do diagnóstico, foram considerados como gengivite os indivíduos que apresentaram pelo menos um sítio com sangramento à sondagem ${ }^{16} \mathrm{e}$ com periodontite os indivíduos que apresentaram pelo menos um sítio com profundidade de sondagem $\geq 4 \mathrm{~mm}$ e perda de inserção clínica $\geq 4 \mathrm{~mm}$, no mesmo sítio ${ }^{17}$.

$N$ as entrevistas com os diabéticos, foram coletados dados relativos ao atendimento na unidade, condições socioeconômicas e comportamentais. $N$ as entrevistas com os 26 gerentes das unidades estudadas, foram coletados os dados relativos à existência de grupos operativos na UBS, partici pação dos profissionais da saúde nos grupos, frequência de participação do dentista, encaminhamento deindivíduos com DM ao serviço odontológico, prioridade para atendimento 
do paciente com DM na saúde bucal e forma de organização dos prontuários nas UBS.

Os resultados do estudo foram digitados e organizados em um banco de dados utilizandoseo softwareStatistical Packagefor Social Science (SPSS) versão 12.0. A análise descritiva dos dados epidemiológicos baseou-se em cálculo de proporções e medidas de tendência central para as variáveis estudadas. Os resultados das entrevistas foram apresentados em percentuais e foram discutidos a partir dos sentidos de integralidade descritos por $\mathrm{M}$ attos ${ }^{1}$.

\section{Resultados}

Não se organizam políticas e serviços em saúde sem conhecer o problema que deverá ser enfrentado. Neste sentido, o estudo teve início com a descrição do perfil do pacientecom DM atendido pelo SUS em Belo H orizonte. Participaram do estudo trezentos indivíduos cuja idade apresentou média de 55,3 ( $\pm 10,0)$, com mediana em 55 anos. Os resultados que caracterizam a amostra estudada se encontram dispostos na Tabela 1.

Tabela 1. Caracterização da amostra. Belo Horizonte (M G), 2006.

\begin{tabular}{|c|c|c|}
\hline Variáveis & $\mathrm{N}$ & $\%$ \\
\hline \multicolumn{3}{|l|}{ Gênero } \\
\hline Masculino & 120 & $40 \%$ \\
\hline Feminino & 180 & $60 \%$ \\
\hline \multicolumn{3}{|l|}{ Idade } \\
\hline 30-54 anos & 148 & $49,3 \%$ \\
\hline Acima de 55 anos & 152 & $50,7 \%$ \\
\hline \multicolumn{3}{|l|}{ Estado civil } \\
\hline Sem companheiro & 128 & $42,7 \%$ \\
\hline Com companheiro & 172 & $57,3^{\circ}$ \\
\hline \multicolumn{3}{|l|}{ Renda } \\
\hline Até 400 reais & 150 & $50 \%$ \\
\hline Acima de 401reais & 150 & $50 \%$ \\
\hline \multicolumn{3}{|l|}{ Escolaridade } \\
\hline Superior e médio & 48 & $16 \%$ \\
\hline Fundamental e sem estudo & 252 & $84 \%$ \\
\hline \multicolumn{3}{|l|}{ Tipo de diabetes melito } \\
\hline Tipo 1 & 86 & $28,7 \%$ \\
\hline Tipo 2 & 214 & $71,3 \%$ \\
\hline \multicolumn{3}{|l|}{ Tabagismo } \\
\hline Sim & 46 & $15,3 \%$ \\
\hline Não & 254 & $84,7 \%$ \\
\hline \multicolumn{3}{|l|}{ Dentes perdidos } \\
\hline Até 12 dentes & 153 & $51 \%$ \\
\hline Acima de 12 dentes & 147 & $49 \%$ \\
\hline
\end{tabular}

Entre os diabéticos avaliados, 9,7\% apresentaram o periodonto saudável, $55 \%$ apresentaram gengivite e $35,3 \%$, periodontite, ou seja, $90,3 \%$ da amostra necessitava de algum tipo de intervenção.

Os dados relacionados ao atendimento odontológico e à organização do programa para diabéticos, na UBS, estão relatados nas tabelas 2 e 3 , respectivamente.

Tabela 2. Distribuição da amostra quanto ao atendimento odontológico recebido na UBS. Belo Horizonte (M G), 2006.

\begin{tabular}{lrr}
\hline & $\mathrm{N}$ & $\%$ \\
\hline Atendimento pelo CD na época do estudo & & \\
Não & 218 & $72,7 \%$ \\
Sim & 82 & $27,3 \%$ \\
Consultas com o dentista no último ano & & \\
$\quad$ Foi ao dentista & 115 & $38,9 \%$ \\
$\quad$ Não foi & 185 & $61,1 \%$ \\
Local de atendimento & & \\
$\quad$ Unidade básica de saúde & 52 & $63,4 \%$ \\
$\quad$ Referência especializada & 3 & $3,6 \%$ \\
Outros & 27 & $33 \%$ \\
\hline
\end{tabular}

Tabela 3. Organização das atividades do programa para diabéticos na UBS. Belo H orizonte (MG), 2006.

\begin{tabular}{lrr}
\hline & N & $\%$ \\
\hline Presença de grupos operativos & & \\
Sim & 24 & $92,3 \%$ \\
Não & 2 & $7,7 \%$ \\
Profissionais & & \\
$\quad$ Dentista & 8 & $33,3 \%$ \\
$\quad$ Nutricionista/psicólogo & 2 & $8,4 \%$ \\
Fisioterapeuta & 6 & $25 \%$ \\
Assistente social & 3 & $12,5 \%$ \\
Participação do dentista & & \\
Participação contínua & 3 & $12,5 \%$ \\
Participação eventual & 5 & $20,8 \%$ \\
Prontuário & & \\
$\quad$ Separado & 20 & $76,9 \%$ \\
Ú nico & 6 & $23,1 \%$ \\
Encaminhamento à saúde bucal & & \\
Sim & 7 & $26,9 \%$ \\
Não & 5 & $19,2 \%$ \\
Eventualmente & 14 & $53,9 \%$ \\
Prioridade de atendimento na saúde bucal & & \\
Sim & 10 & $38,5 \%$ \\
Não & 4 & $15,3 \%$ \\
Eventualmente & 12 & $46,2 \%$ \\
Total & 26 & 100 \\
& &
\end{tabular}


Em relação à prioridade de atendimento ao diabético, em $61,5 \%$ das UBS, ela existesomente em caso de "necessidade" ou determinada urgência.

\section{Discussão}

A expressiva prevalência degengivite( $55 \%$ ) epe riodontite (35,3\%) no grupo estudado já se configurava como hipótese neste estudo. A literatura tem mostrado queindivíduos diabéticos apresentam maior prevalência de doenças gen givaise periodontais ${ }^{18-23}$, quando comparados à população geral. Os dados do SB-BRASI L24, inquérito epidemiológico de abrangência nacional, mostram um prevalência de gengivite na população brasileira de 9,97\% em indivíduos entre 35 e 44 anos e 3,27\% em indivíduos entre 65 e 74 anos. Em relação à periodontite, a prevalência de indivíduos com bolsas periodontais acima de $4 \mathrm{~mm}$ foi de 1,34 para a faixa etária de quinze a dezenove anos, 9,98\% na população de 35 a 44 anos e $6,3 \%$ nos indivíduos de 65 a 74 anos. Considerando que os critérios de exame neste estudo e no SB-BRASI L ${ }^{24}$ foram os mesmos, os dados confirmam a gravidade da saúde periodontal deste grupo de diabéticos.

Outros estudos também mencionam que os portadores de diabetes apresentam percentuais mais elevados de bolsas profundas e perdas graves de inserção periodontal do queos indivíduos não diabéticos ${ }^{25}$; que adultos diabéticos apresentam maior gravidade das condições periodontais quando comparados aos não diabéticos e que estes apresentam três vezes mais chance de sofrer perda detecido periodontal de suporte ${ }^{26,27}$.

A pesar desta gravidade, 0 exame periodontal parece não fazer parte integrante da rotina da maioria dos profissionais do serviço público ou privado. 0 quese observa frequentementeéuma profissão que se organizou em torno de uma patologia - a cárie dentária - e tem na prótese, que deveria ser a marca do fracasso da odontologia (resultado da perda dentária), um "totem e marco distintivo da profissão" 28. Procedimentos para controle e tratamento da DP vão sendo relegados a segundo plano ${ }^{29}$.

Este fato é demonstrado quando, apesar do padrão epidemiológico apresentado, somente $27,3 \%$ dos diabéticos relataram estar em atendimento odontológico na U BS à época do estudo e apenas 38,9\% haviam procurado o dentista no último ano. Considerando que a gengivite tem grandes chances de ser controlada, por meio de procedimentos incluídos no rol de atividades da
U BS (atenção básica), 55\% dos diabéticos deveriam estar em tratamento. Entreos diabéticos examinados, 35,3\% apresentaram periodontite mas somente $3,6 \%$ dos entrevistados estavam em tratamento na clínica odontológica especializada.

No entanto, todos os incluídos no estudo eram cadastrados no HIPERDIA, indicando que a grande maioria dos indivíduos diabéticos estava sendo atendida periodicamente pelos médicos das unidades.

Entre as atividades previstas pelo M inistério da Saúde ${ }^{7}$, encontra-se a formação de grupos operativos, sobretudo no caso de doenças crônicas como a DM , como forma de vinculação com a U BS e desenvolvimento de programas educativos para controle da doença e promoção de saúde. Através das entrevistas com os gerentes, observou-se que $92,3 \%$ das unidades realizavam trabalho com grupos voltados ao diabético. Dentre os profissionais da saúde, excetuando-se os médicos e enfermeiros, que participam efetivamente destes grupos, o dentista seria o mais presente $(33,3 \%)$, sendo que $12,5 \%$ apresentavam uma participação contínua e 20,8\%, uma colaboração quando solicitado. Deste modo, a participação da odontologia no atendimento ao diabético é incipiente, em todas as fases nas quais se investe no controle da doença.

Acredita-se que a participação dos dentistas nos grupos operativos melhoraria de maneira significativa a prevalência degengivitenos diabéticos (55\%). M edidas simples como a orientação e a motivação a estes indivíduos, por exemplo, representariam melhorias das condições gengivais. Para Tinoco e Tinoco ${ }^{29}$, um indivíduo motivado em relação à sua saúde periodontal tem mais chances dese beneficiar das medidas deprevenção. Como o grau de motivação oscila com o passar do tempo, seria importante a presença do dentista nestes grupos, com mai or frequência.

A pesar da importância do trabal ho em equipe, outros profissionais são ainda menos presentes nos trabal hos dos grupos operativos. $N$ as entrevistas com os diabéticos, apenas 3,4\% dos entrevistados relataram receber atendimento com outros profissionais, como nutricionista, fisioterapeuta, psicólogo e assistente social. Ressalte-se que estes profissionais são escassos na maioria das equipes, em Belo Horizonte.

Quanto ao encaminhamento dos diabéticos para consulta odontológica, em $73,1 \%$ das UBS, constatou-se que o indivíduo diabético não era encaminhado para tratamento na saúde bucal como rotina mas apenas em casos de "necessidade" ou urgência. 0 mesmo critério era adota- 
do para priorizar 0 atendimento do diabético, em $61,5 \%$ das UBS.

Deste modo, 0 atendimento odontológico é completamente desvinculado do atendimento feito pela equipe do PSF (medico e enfermeiro). Os cuidados em saúde que deveriam ser planejados interdisciplinarmente têm sido realizados isoladamente, por cada profissional. Somente $23,1 \%$ das UBS trabalham com prontuário único.

Considerando a integralidade da atenção ao diabético, o primeiro sentido descrito por $M$ attos $^{1}$ aborda a visão integral do profissional de saúde em relação ao usuário do serviço. A atitude de um profissional de saúde frente a um paciente que traz uma necessidade seria buscar conhecer todosos aspectosqueen volvem a sua vida, em prol da construção de uma imagem mais ampla desse campo de necessidades, permitindo que as ações a serem defini das contemplem fatores de risco à saúde e condições outras que não relacionadas somente ao motivo da consulta ${ }^{1}$. Dentro desta perspectiva, o ideal seria que nas UBS os profissionais envolvidos no atendimento dos usuários pudessem ter esta visão, mas, no entanto, percebe-seque na prática isto não ocorre. Os indivíduos com DM atendidos pelo médicos sequer eram encaminhados para atendimento odontológico. Isto evidencia a grande dificuldade em perceber até as necessidades de saúde dos indivíduos como um todo. Baldini et al. ${ }^{30}$ afirmam que o fato da odontologia não estar presente desde o início do programa PSF possivelmente acarretou prejuízos no processo de integralização dos profissionais correlacionados. Outro aspecto que merece ser evidenciado é que o pequeno número de psicólogos, fisioterapeutas, nutricionistas e assistentes sociais, na rede municipal de Belo Horizonte, pode estar comprometendo a organização de uma equipe de saúde mais efetiva e com maior clareza das necessidades globais dos usuários.

O segundo sentido da integralidade a ser considerado na análise se refere às formas de organizar as práticas e processos de trabalho em saúde ${ }^{1}$. Os resultados encontrados mostram ainda que existe pouca integração entre os profissionais das unidades de saúde. Dentre as pessoas estudadas, a grande maioria - 218 (72,7\%) - não estava sob tratamento odontológico, $61,1 \%$ não haviam sido atendidos pelo dentista, no último ano, embora $96,6 \%$ realizassem controle médico periódico. 0 pequeno número de indivíduos atendidos pelo dentista (27,3\%) pode retratar uma falta de integração das ações médicas e odontológicas, visto que quase todas as unidades do município pos- suem dentista. 0 trabalho em equipe é fundamental, uma vez que os dados relatados na literatura nacional e internacional, bem como os descritos neste estudo, apontam para uma maior prevalênciaegravidade degengivitee periodontite em indivíduos com DM quando comparados à prevalência da doença na população geral24. Juntando-se a estes dados o fato da doença periodontal poder dificultar o controleda glicemia, reafirma-se a necessidade de serem revistas as práticas e processos de trabalho em saúde no sentido de enfatizar o trabalho interdisciplinar no controle da glicemia dos diabéticos.

Considerando-se o pequeno número de unidades que referenciam os indivíduos diabéticos para tratamento odontológico - 26,9\% - e ainda os $73,1 \%$ das unidades que não 0 encaminham regularmente, pode-se supor que exista um desconhecimento da equipe de saúde sobre a necessidade de atendimento odontológico ao indivíduo com DM ou uma dificuldade de acesso ao serviço odontológico que faz com que o indivíduo seja encaminhado apenas quando apresenta um quadro de urgência. Outra questão a ser abordada é que os profissionais da saúde apresentam uma concepção compartimental izada do conhecimento de cada área e, ainda que atuem no mesmo espaço de trabalho, a prática permanececentrada no núcleo de conhecimento de cada trabalhador, ocasionando um desencontro dos saberes ${ }^{31}$. Os resultados apresentados mostram que nas UBS o trabalho tem sido organizado de forma fragmentada, sem a participação efetiva e o envolvimento de outros profissionais da área da saúde, que são fundamentais ao desenvolvimento da prática profissional. A própria divisão do prontuário em médico eodontológico retrata esta segmentação. N este estudo, apenas $23,1 \%$ das unidades de saúde da família utilizam prontuários únicos para os indivíduos. 0 trabalho em equipeinterdisciplinar éuma potencial ferramenta de mudança nos processos de trabalho, alicerçando a integralidade e seus dispositivos (acolhimento, vínculo, autonomia, resolubilidade, responsabilização) ${ }^{32}$. É um caminho que deve ser almejado pelas equipes de saúde.

Em relação à integração entre os diferentes níveis de atenção, terceiro sentido da integralidadeutilizado nesta análise ${ }^{1}$, observa-se um pequeno número de diabéticos atendidos na atenção básica $(27,3 \%)$ e atenção especializada (3,6\% ) no SU S/BH . No que concerneà aten ção básica, 0 número encontrado - 27,3\% - podemostrar uma dificuldade da saúde bucal em responder as necessidades da população no âmbito da atenção 
primária. Como a assistência odontológica pública no Brasil tem se restringido quase completamente aos serviços básicos, ainda assim, com grande demanda reprimida ${ }^{33}$, para que 0 grau de resolutividade neste nível de atenção básica seja satisfatório para os diabéticos, ou seja, os casos com gengivite ( $55 \%$ ) sejam solucionados, deve se tornar rotina a utilização adequada dos meios disponíveis de diagnóstico no exame periodontal ${ }^{29}$, coordenada com procedimentos decontrole e prevenção desta doença, que incluem o trabalho nos grupos operativos. É claro que a prática odontológica apresenta certas especificidades, uma vez queinclui num mesmo ato o diagnóstico e a terapêutica cirúrgica, cuja conclusão pode se arrastar por semanas ou meses. Esta característica do ato odontológico introduz um complicador a mais quando se discute acesso a serviços, que é a combinação de uma alta prevalência de doenças (e, consequentemente, de necessidades), com uma lógica de tratamento complexa, demorada e onerosa ${ }^{32}$.

A Política Nacional de Saúde Bucal, Brasil Sorridente, reúne uma série de ações em saúde bucal, voltadas para cidadãos de todas as idades. Até seu lançamento, em 2004, apenas 3,3\% dos atendimentos odontológicos feito no SUS correspondiam a tratamentos especializados. A quase totalidade era de procedimentos mais simples, como extração dentária, restauração e pequenas cirurgias ${ }^{34}$. Dado muito semelhante ao encontrado neste estudo, em que apenas $3,6 \%$ do atendimento ao diabético era ofertado pela rede secundária. Este valor não corresponde às necessidades de atendimento dos indivíduos com DM, uma vez que estes podem ter suas condições de saúde bucal agravadas e, portanto, necessitarem deatendimento especializado. É evidentea baixa capacidade de oferta dos serviços de atenção secundária eterciária, comprometendo, em conse quência, o estabelecimento de adequados siste mas de referência e contrarreferência em saúde bucal na quase totalidade dos sistemas locorregionais de saúd $e^{34}$. A expansão da rede assistencial de atenção secundária e terciária não acompanhou, no setor odontológico, o crescimento da oferta de serviços de atenção básica ${ }^{34}$.

N esse sentido, a política Brasil Sorridente propõe garantir as ações de promoção, prevenção e recuperação da saúde bucal dos brasileiros. No âmbito da assistência, estas diretrizes apontam para a ampliação e qualificação da aten ção básica, possibilitando 0 acesso a todas as faixas etárias e a oferta de mais serviços, assegurando atendimento no nível secundário, de modo a buscar a integralidade da atenção. Os centros de especialidades odontológicas estão preparados para oferecer à população os serviços de diagnóstico bucal, periodontia especializada, cirurgia oral menor, endodontia e atendimento a portadores de necessidades especiais ${ }^{34}$.

A organização das instituições na busca da integração dos cuidados em saúde, em todo o sistema, através da conformação de uma rede de serviços estruturada de forma a maximizar as possi bilidades de atendimento, é de fundamental importância. Também a articulação entre os diferentes níveis, com a estruturação de sistemas de referência econtrarreferência que dêem conta das necessidades destes indivíduos, bem como a ação interdisci plinar, tornam-se imperiosas para seguir-se os caminhos rumo à integralidade da atenção ${ }^{35}$.

\section{Conclusões}

Foi observada uma prevalência significativa de gengivitee periodontite na população de diabéticos estudada, $55 \%$ e $35,3 \%$, respectivamente, 0 que torna a atenção à saúde bucal de grande importância, tanto para a melhoria das condições de saúde bucal quanto para o controle da glicemia para os indivíduos diabéticos. Em relação à rotina de atendimento do indivíduo diabético no SU S/BH, com vistas à integralidade da atenção, pôde-se observar, através das entrevistas com os diabéticos, uma participação incipiente deoutros profissionais da saúde, além do médico, no atendimento a estes indivíduos (3,4\%). Apesar de 0 dentista estar presente em quase todas as UBS, 0 acesso a este ainda é restrito e apenas 27,3\% dos indivíduos estudados estavam sob tratamento odontológico na atenção básica, 3,6\% na atenção especializada eapenas 38,9\% haviam procurado 0 atendimento odontológico no último ano. Em relação ao desenvolvimento de grupos operativos com vistas à prevenção e controle do diabetes, pôde-se verificar, através das entrevistas com os gerentes, que a maioria das unidades de saúdeestudadas real izavam estes grupos (92,3\%). Em 33,3\% destas, o dentista participava dos grupos, sendo sua presença vinculada geral mente ao convite da equipe. Em 73,1\% das unidades, os indivíduos diabéticos não eram encaminhados para a odontologia ou o eram em caso de alguma necessidade/dor. Em $61,5 \%$ das unidades, 0 diabético não tinha prioridade no atendimento na saúde bucal ou a tinha em caso de necessidade/dor. Outros profissionais, como nutricionis- 
tas, fisioterapeutas, psi cólogos e assistentes sociais, participavam das atividades dos grupos em $4,2 \%, 25 \%, 4,2 \%$ e $12,5 \%$ das unidades estudadas, respectivamente. Outro aspecto relevante é que, na maioria das UBS (76,9\%), o prontuário da medicina e da odontologia eram separados, 0 que reforça a hipótese de existir pouca integração entre os profissionais nas equipes. A atenção integral ao pacientediabético, garantindo 0 atendimento em todos os níveis, bem como a integração dos profissionais dentro da própria unidade de saúde, são fatores essenciais para contemplar a integralidade das ações em saúde no atendimento ao indivíduo com DM .

\section{Colaboradores}

Silva AS, Vargas AMD e Ferreira EF participaram da elaboração do artigo nas fases de concepção, delineamento, análises e redação do artigo. Abreu M H NG participou da fase de análisee interpretação dos dados.

\section{Referências}

1. M attos RA. Os sentidos da integralidade: algumas reflexões acerca de valores que merecem ser defendidos. In: Pinheiro R, M attos RA, organizadores. Os sentidos da integralidade na atenção e no cuidado à saúde. Rio de Janeiro: Instituto de M edicina Social, Universidade do Estado do Rio de Janeiro/ABRASCO; 2001. p. 39-64.

2. M attos RA. Integralidade e a formulação de políticas específicas de saúde. In: Pinheiro R, M attos RA, organizadores. Construção da integralidade: cotidiano, saberes e práticas em saúde. Rio de Janeiro: Instituto de M edicina Social, Universidade do Estado do Rio de Janeiro/ABRASC0; 2003. p. 45-60.

3. Sá PK. Integralidade da Atenção no Programa M édico de Família de Niterói - RJ [dissertação]. Rio de Janeiro (RJ): Escola Nacional de Saúde Pública, Fundação Oswaldo Cruz; 2003.

4. Campos CEA. O desafio da integralidade segundo as perspectivas da vigilância da saúde e da saúde da família. Cien Saude Colet 2003; 8(2):509-584.

5. Mattos RA. A integralidade na prática (ou sobre a prática da integralidade). Cad Saude Publica 2004; 20:1411-1416.

6. Franco TB, Bueno WS, M erhy EE. O acolhimento e os processos de trabalho em saúde: o caso de Betim, M inas Gerais. Cad Saude Publica 1999; 15:345-353.

7. Brasil. Ministério da Saúde. Secretaria de Ações Programáticas e Estratégicas. Plano de reorganização da atenção à hipertensão arterial e ao diabetes mellitus. [site da Internet] [acessado 2007 mar 05]. Disponível em: http://dtr2004.saude.gov.br/dab/ hipertensaodiabetes/documentos/publicacoes/ miolo2002.pdf 
8. Brasil. M inistério da Saúde. Secretaria de Políticas de Saúde. Departamento de Ações Programáticas Estratégicas. Plano de reorganização da atenção à hipertensão arterial e ao diabetes mellitus: hipertensão arterial e diabetes mellitus. [Série C. Projetos, Programas e Relatórios n 59]. Brasília: Ministério da Saúde; 2001.

9. Oliver RC, Tervonen T. Diabetes: A risk factor for periodontitis in adults. J Periodontol 1994; 65:530538.

10. Miller LS, Manwell MA, Newbold D, Reding ME, Rasheed A, Blodgett J, Kornman KS. The relationship between reduction in periodontal inflammation and diabetes control: a report of 9 cases. J Periodontol 1992; 63:843-848.

11. Brasil. Conselho Nacional de Saúde. Resolução no 196 de 10 de outubro de 1996. Aprova diretrizes e normas regulamentadoras de pesquisas envolvendo seres humanos. Bioética 1996;4(2):15-25.

12. Lwanga SK, Lemeshow S. Sample size determination in health studies: a practical manual. Geneva: WHO; 1991.

13. Sonis ST, Fazio RC, Fang LF. Diabetes Melito. In: Teles JCB, M oreira LC, Souza RA, organizadores. Princípios e Prática de M edicina Oral. Rio de Janeiro: Guanabara Koogan; 1996. p. 115-127.

14. Brasil. Ministério da Saúde. HIPERDIA: sistema de cadastramento e acompanhamento de hipertensos e diabéticos. [site da Internet] [acessado 2007 mar 05]. Disponível em: http://hiperdia.datasus.gov.br
15. Brasil. Ministério da Saúde. Agência Nacional de Vigilância Sanitária. Serviços O dontológicos: Prevenção e Controle de Riscos. [Série A. N ormas e Manuais Técnicos] Brasília: M inistério da Saúde; 2006.

16. Lang N P, Adler R, Joss A, Nyman $S$. Absence of bleeding on probing: a indicator of periodontal stability. J Clin Periodontol 1990; 17:714-721.

17. American Academy of Periodontology. Parameters of care. J Periodontol 2000; 71(5 Suppl):847-883.

18. Barilli ALA, Passos ADC, M arin-N eto JA, Franco LJ. Periodontal disease in patients with ischemic coronary atherosclerosis at a University Hospital. Arq Bras Cardiol 2006; 87:695-700.

19. Khader YS, Dauod AS, El-Qaderi SS, Alkafajei A, Batayha WQ. Periodontal status of diabetics compared with nondiabetics: a meta-analysis. J Diabetes Complications 2006; 20:59-68.

20. Drumond-Santana T, Costa FO, Zenóbio EG, Soares RV, Santana TD. Impacto da doença periodontal na qualidade de vida de indivíduos diabéticos dentados. Cad Saude Publica 2007; 23: 637-644.

21. Moore PA, Weyant RJ, Mongelluzzo M B, Myers DE, Rossie K, Guggenheimer J, Block HM, Huber $H$, Orchard T. Type 1 diabetes mellitus and oral health assessment of periodontal disease. J Periodontol 1999; 70:409-417.

22. Cianciola LJ, Park BH, Bruck E, Mosovich L, Genco RJ. Prevalence of periodontal disease in insulindependent diabetes mellitus. J Am Dent Assoc 1982; 104:653-60. 
23. Löe $\mathrm{H}$. Periodontal disease: the sixth complication of diabetes mellitus. Diabetes Care 1993; 16:329 334.

24. Brasil. M inistério da Saúde. Projeto SB-Brasil 2003. Condições de Saúde bucal da População Brasileira 20022003: Resultados Principais. [site da Internet] [acessado 2007 mar 04]. Disponível em: http://www.cfo. org.br/download/pdf/relatorio_sb_brasil_2003.pdf

25. Tomita NE, Chinellato LEM, Pernambuco RA, Lauris JRP, Franco LJ. Condições periodontais e diabetes mellitus na população nipo-brasileira. Rev Saude Publica 2002; 36:607-613.

26. Papapanou PN, Lindhe J. Epidemiologia da doença periodontal. In: Lindhe J, organizador. Tratado de periodontia clínica e implantologia oral. Rio de Janeiro: Guanabara Koogan; 1999. p. 43-65.

27. Papapanou PN. World Workshop in Clinical Periodontics. Periodontal diseases: epidemiology. Ann Periodontol 1996; 1:1-36.

28. Botazzo C. Saúde bucal e cidadania: transitando entre a teoria e a prática. In: Pereira AC, organizador. Odontologia em saúde coletiva: planejando ações e promovendo saúde. Porto Alegre: Artmed; 2003.

29. Tinoco EM B, Tinoco NMB. Diagnóstico e prevenção das doenças periodontais. In: Buischi IP, organizador. Promoção da Saúde Bucal na Clínica Odontológica. São Paulo: Artes M édicas/EAP-APCD; 2000 p. 101-123.

30. Baldani MH, Fadel CB, Possamai T, Queiroz M GS. A inclusão da odontologia no Programa Saúde da Família no Estado do Paraná, Brasil. Cad Saude Publica 2005; 21:1026-1035.

31. Santos AM, Assis M M A. Da fragmentação à integralidade: construindo e (des) construindo a prática de saúde bucal no Programa de Saúde da Família (PSF) de Alagoinhas, BA. Cien Saude Colet 2006; $11(1): 53-61$
32. Mueller CD, Schur CL, Paramore LC. Access to dental care in the United States: estimates from a 1994 survey. JADA 1998; 129:429-437.

33. Brasil. M inistério da Saúde. Secretaria de Atenção à Saúde. Departamento de Atenção Básica. Coordenação Nacional de Saúde Bucal. Diretrizes da Política Nacional de Saúde Bucal. [site da Internet] [acessado 2007 mar 05]. Disponível em: http:// dtr2004.saude.gov.br/dab/saudebucal/publicacoes/ diretrizes_da_politica_nacional_de_saude_bucal.pdf

34. Brasil. M inistério da Saúde. Secretaria de Atenção à Saúde. Departamento de Atenção Básica. Coordenação Nacional de Saúde Bucal. Brasil Sorridente. [site da Internet] [acessado em 2007 mar 03]. Disponível em: http://dtr2004.saude.gov.br/dab/ saudebucal/brasil_sorridente.php

35. Alves VS. Um mōdelo de educação em saúde para o Programa Saúde da Família: pela integralidade da atenção e reorientação do modelo assistencial. Interface (Botucatu) 2005; 9(16):39-52.

Artigo apresentado em 30/11/2007

Aprovado em 12/01/2008

Versão final apresentada em 04/03/2008 